\title{
Safety and efficacy of personal care products containing colloidal oatmeal
}

This article was published in the following Dove Press journal:

Clinical, Cosmetic and Investigational Dermatology

7 November 2012

Number of times this article has been viewed

\section{Maryline Criquet ${ }^{\prime}$ \\ Romain Roure' \\ Liliane Dayan² \\ Virginie Nollent' \\ Christiane Bertin'}

'Johnson \& Johnson Santé Beauté France, Issy les Moulineaux, ${ }^{2}$ Independent consultant dermatologist, Paris, France
Correspondence: Christiane Bertin Johnson \& Johnson Santé Beauté France, I, rue Camille Desmoulins, 92787 Issy-les-Moulineaux, France Tel +33I 55004733

Email cbertin@its.jnj.com
Background: Colloidal oatmeal is a natural ingredient used in the formulation of a range of personal care products for relief of skin dryness and itchiness. It is also used as an adjunctive product in atopic dermatitis. The safety of personal care products used on vulnerable skin is of particular importance and the risk of developing further skin irritations and/or allergies should be minimized.

Methods: In a series of studies, we tested the safety of personal care products containing oatmeal (creams, cleansers, lotions) by assessing their irritant/allergenic potential on repeat insult patch testing, in safety-in-use and ocular studies using subjects with nonsensitive and sensitive skin. We also tested the skin moisturizing and repair properties of an oatmeal-containing skin care product for dry skin.

Results: We found that oatmeal-containing personal care products had very low irritant potential as well as a very low allergenic sensitization potential. Low-level reactions were documented in $1.0 \%$ of subjects during the induction phase of repeat insult patch testing; one of 2291 subjects developed a persistent but doubtful low-level reaction involving edema during the challenge phase in repeat insult patch testing. No allergies were reported by 80 subjects after patch testing after in-use application. Sustained skin moisturizing was documented in subjects with dry skin that lasted up to 2 weeks after product discontinuation.

Conclusion: Our results demonstrate that colloidal oatmeal is a safe and effective ingredient in personal care products. No allergies were reported by consumers of 445,820 products sold during a 3-year period.

Keywords: Avena sativa, colloids, protective agents, atopic dermatitis, irritant dermatitis, allergenic dermatitis, skin care

\section{Introduction}

Colloidal oatmeal is a natural product derived from oat grains (Avena sativa) that have been ground into a very fine powder, with a complex chemical composition including polysaccharides, lipids, proteins, flavonoids, minerals, and vitamins. ${ }^{1}$ Colloidal oatmeal is appreciated for its moisturizing, cleansing, antioxidative, and anti-inflammatory properties, which are conferred by its chemical heterogeneity. A variety of oatmeal-containing personal care products are available, including bath products, shampoos, moisturizers, and shaving foams, for the protection and alleviation of, eg, rashes and dry skin, and for cleansing and moisturizing. ${ }^{2}$ Used as adjunctive therapy in infants with moderateto-severe atopic dermatitis, it can help to reduce the need for high-potency topical corticosteroids. ${ }^{3}$ The anti-irritant effects of colloidal oatmeal appear to be mediated by avenanthramides, which inhibit immune-dependent skin inflammation. ${ }^{4}$ 
Epidemiological studies have shown that a high number of individuals suffer from sensitive skin, with a prevalence of $51 \%-52 \%$ in women and $38 \%$ in men. ${ }^{5,6}$ Symptoms of cosmetic-induced skin discomfort, such as burning, stinging, and itching, are reported more commonly by individuals with sensitive skin than by those who consider themselves nonsensitive. ${ }^{6}$ Fragrances and preservatives are the most frequently identified allergenic sensitizers in cosmetic dermatitis. ${ }^{7}$ The inclusion of food proteins in personal care products is controversial in terms of whether topical application of oatmeal-containing products induces percutaneous sensitization in subjects with atopic dermatitis and to what extent. ${ }^{8-10}$

Therefore, it is of the utmost importance that personal care products, in particular those intended to treat sensitive skin or to help in the treatment of atopic dermatitis, do not aggravate existing skin conditions and that the risk of allergic reactions is minimized by excluding sensitizing ingredients. We sought to determine the irritant and allergenic potential of a range of personal care products containing colloidal oatmeal as an active ingredient after repeated applications in human subjects including those with sensitive skin or a history of atopic dermatitis. We also assessed the efficacy of an oatmeal-containing cream in relieving skin dryness.

\section{Safety assessment}

\section{Repeat insult patch tests}

Twelve independent studies were performed at two centers in two countries (10 studies in the US, and two studies in Romania) between February 2000 and May 2009. The tests were conducted under the supervision of a dermatologist who participated in the evaluation of irritation/allergic reactions to the test materials. Each panel comprised 114-245 male and female volunteers who gave their written informed consent before enrolment. Subjects with dermatological or other medical or physical conditions precluding topical application of the test material, were excluded, along with pregnant and nursing women. The study centers used different protocols. For the induction period, a series of nine induction patchings were performed over a period of 3 weeks.

The materials tested were 12 skin care products containing oatmeal as the active ingredient. These comprised three lotions, two face creams, one serum product, two cleansing lotions, one exfoliating cleanser, two baby products (one cream and one cleanser), and one hand cream.

At the US site, an occlusive or semiocclusive patch containing $0.2 \mathrm{~g}$ of test material was applied to the left side of the back where it remained for 24 hours. Subjects were instructed to keep the patch as dry as possible and to remove it after 24 hours. No test material was applied for the following 24 (on weekdays) or 48 hours (on weekends), after which evaluation for potential dermal reactions was undertaken. Reactions after patching were scored according to a modified version of the International Contact Dermatitis Research Group scoring system ${ }^{11}$ (see Table 1).

At the Romanian site, patches containing $25 \mu \mathrm{L}$ of the test material were applied to the back in a semiocclusive manner and removed after 48 hours. The dermal response during this period was scored using a five-point scale (see Table 1). The rest period comprised 2 weeks without application of the test material. During the challenge period, patches were prepared and fixed in the same manner as in the induction period, but on the right side of the back (ie, a virgin site).

The patches were removed after 24 hours at the US study center, and scoring of skin reactions was performed in the same manner as before at 24, 48, 72, and 96 hours after patching. At the Romanian study center, the patches remained in place for 48 hours and skin reactions were scored 30 minutes and 24 and 48 hours after patch removal using the International Contact Dermatitis Research Group scoring system.

Table I Scoring methods used for evaluation of irritation/allergic skin reaction

\begin{tabular}{|c|c|}
\hline Grade & Description \\
\hline \multicolumn{2}{|c|}{ US studies: induction period and challenge period } \\
\hline \multicolumn{2}{|c|}{ (ICDRG-modified scoring scale for dermatitis' $\left.{ }^{\prime \prime}\right)$} \\
\hline 0 & No visible reaction \\
\hline \pm & Faint, minimal erythema \\
\hline I & Erythema \\
\hline 2 & Intense erythema, induration \\
\hline 3 & Intense erythema, induration, vesicles \\
\hline 4 & $\begin{array}{l}\text { Severe reaction with erythema, induration, } \\
\text { vesicles, pustules (including weeping) }\end{array}$ \\
\hline E & Indicates presence of edema \\
\hline \multicolumn{2}{|c|}{ Romanian studies: induction period } \\
\hline- & No evidence of any effect \\
\hline I & Mild (pink, uniform erythema) \\
\hline 2 & Moderate (pink-red erythema) \\
\hline 3 & $\begin{array}{l}\text { Marked (bright red erythema with/without } \\
\text { petechiae or papules) }\end{array}$ \\
\hline 4 & $\begin{array}{l}\text { Severe (deep red erythema with/without } \\
\text { vesiculation or weeping) }\end{array}$ \\
\hline \multicolumn{2}{|c|}{ Romanian studies: challenge period (ICDRG scoring scale) } \\
\hline- & Negative \\
\hline$+?$ & Doubtful reaction (slight erythema) \\
\hline+ & Weak reaction (nonvesicular reaction) \\
\hline++ & Strong reaction (erythema, edema, and/or vesicles) \\
\hline+++ & Severe reaction with blisters \\
\hline
\end{tabular}

Abbreviation: ICDRG, International Contact Dermatitis Research Group System. 


\section{Safety-in-use tests}

Twelve independent studies were performed at four study centers in four countries (seven studies in the UK, two in Poland, two in Germany, and one in Bulgaria) between April 2006 and August 2009. Subjects with a range of self-reported skin types and sensitivities were recruited. In the UK studies, the test materials were applied for 7, 10 or 28 days and skin reactions were evaluated using self-assessment questionnaires (adverse reaction, yes/no, severity slight, moderate, severe). The studies conducted in Poland involved a 3-week application period, with ophthalmological assessment by slit-lamp and evaluation of subjective functional signs before and after use on day 21 and subjective self-assessment patient questionnaires regarding functional/physical signs. The Bulgarian study involved 3 weeks of application, with dermatological evaluation (detection of allergy, irritation, dryness, discomfort, pimples) and subject self-assessment questionnaires (adverse reaction, yes/no). The studies in Germany included a 4-week application period in adults and children followed by occlusive patch testing in the adult participants. Reactions during the application period and after patch testing were evaluated by a dermatologist-allergologist at 24, 48, and 72 hours after patching. The following test materials were used: shower and bath oil, cream, moisturizing oil, shower gel, night cream, conditioning shampoo, body lotion, liquid hand wash, face and eye cleansing lotion (two products), facial exfoliating cleanser, intimate wash, and baby milk.

\section{Efficacy assessment}

An open prospective study was performed to assess the effect of an oatmeal-containing body cream on various aspects of dry skin. Fifty female subjects aged 20-67 years were enrolled. All subjects were of Caucasian origin and had dry to very dry skin of phototype I-IV. The study duration was 6 weeks, which included 4 weeks of study product application followed by 2 weeks without product application. Subjects were instructed to avoid use of other skin care products, with the exception of cleansers, for 3 days before starting the study, as well as for 2 weeks following the study period. The study product was to be applied once a day in the morning on the leg (external part of the calf) and on the inner forearm according to the subjects' usual application habits. Assessments were performed at baseline, and on days 1, 14, and 28, and 2 weeks after the last application (day 42). Skin hydration (moisture content in the upper epidermis) was assessed using a Corneometer ${ }^{\circledR} \mathrm{CM} 825$ (Courage + Khazaka Electronic GmbH, Germany). The mean of three consecutive measurements was calculated. The desquamation index and the surface area of dead epithelial cells were assessed using adhesive disc stripping (D-squame ${ }^{\circledR}$; CuDerm Corporation, Dallas, TX) with subsequent digital image analysis.

Clinical efficacy was assessed by a dermatologist. Assessments included visual examination of skin dryness and appearance of epithelial squamae, as well as tactile evaluation of skin roughness. A $10 \mathrm{~cm}$ visual analog scale was used, where 0 represented "none" and 10 was "severe". Subject self-assessment involved a questionnaire with a five-point scale ranging from 1 ("agree") to 5 ("disagree"). The number of responses from category 1 and 2 (ie, "agree" and "rather agree") was combined for analysis. Measurements were made on the treated body areas (leg and inner forearm), as well as on an untreated area on the mid-thigh which served as a control site. Clinical assessments were performed only on the treated leg and on the control area.

Statistical analysis was performed using SPSS software (SPSS Inc, Chicago, IL). Data at each assessment time point are shown as the mean \pm standard error of the mean. At each time point, data were compared with baseline and, where applicable, the difference between treated and untreated sites was compared, and the difference was expressed as a variation percentage. The Student's paired $t$-test (for normal distribution) or the Wilcoxon test (for nonnormal distribution) was used at a significance level of $P \leq 0.05$. All subjects involved in the study gave their written informed consent before enrolment. All studies described herein were conducted according to the ethical principles outlined in the Declaration of Helsinki and according to good clinical practices.

\section{Results Safety analysis}

Of the 2565 men and women who enrolled in the 12 repeat insult patch testing studies, 274 discontinued for reasons other than a reaction to the test material. In the induction period, a total of 23 patients experienced a reaction. We observed 34 transient low-level grade \pm reactions (ie, faint minimal erythema) in 20 subjects (including one patient with eight consecutive faint erythema readings), six transient low-level grade 1 reactions in six subjects, and mild erythema in one subject. In the challenge period, 17 patients had a reaction. This comprised 18 transient low-level grade \pm reactions in 14 subjects, nine transient low-level grade 1 reactions in seven subjects, and five grade 1 reactions with edema in three subjects (Table 2). Edematous reactions were not confirmed in subsequent patch tests for two subjects. However, for the 
Table 2 Summary of repeat insult patch testing studies

\begin{tabular}{|c|c|c|c|c|}
\hline Test material & Date/country & Participants & Application & Results \\
\hline Lotion & $\begin{array}{l}\text { June-July } 2005, \\
\text { US }\end{array}$ & $\begin{array}{l}245 \text { included/ } \\
207 \text { completed } \\
66 \text { male, } 141 \text { female } \\
\text { Aged } 18-70 \text { years }\end{array}$ & Occlusive & $\begin{array}{l}\text { No reaction during induction phase or challenge phase } \\
\text { Conclusion: no potential for dermal irritation or } \\
\text { sensitization }\end{array}$ \\
\hline Lotion & $\begin{array}{l}\text { December 200I- } \\
\text { January 2002, US }\end{array}$ & $\begin{array}{l}226 \text { included/ } \\
209 \text { completed } \\
55 \text { male, } 154 \text { female } \\
\text { Aged } 18-69 \text { years }\end{array}$ & Occlusive & $\begin{array}{l}\text { Induction phase: one transient low-level } \pm \text { reaction in } \\
\text { one subject (reading I) } \\
\text { Challenge phase: three low-level } \pm \text { reactions in one } \\
\text { subject }(48,72,96) \text {; one level I + edema reaction ( } 72 \text { hours), } \\
\text { one transient low-level reaction (I) in one subject ( } 96 \text { hours) } \\
\text { Remarks: test material did induce an edematous reaction } \\
\text { indicative of dermal sensitization in one human subject } \\
\text { This reaction was not confirmed by a second patch testing } \\
\text { Conclusion: no potential of the product for dermal sensitization }\end{array}$ \\
\hline Lotion SPFI5 & $\begin{array}{l}\text { July-August 200I, } \\
\text { US }\end{array}$ & $\begin{array}{l}221 \text { included/ } \\
193 \text { completed } \\
55 \text { male, } 138 \text { female } \\
\text { Aged } 18-69 \text { years }\end{array}$ & Semiocclusive & $\begin{array}{l}\text { No reaction during induction phase or challenge phase } \\
\text { Conclusion: no potential for dermal irritation } \\
\text { or sensitization }\end{array}$ \\
\hline $\begin{array}{l}\text { Cleansing } \\
\text { lotion }\end{array}$ & $\begin{array}{l}\text { February-April 2005, } \\
\text { US }\end{array}$ & $\begin{array}{l}227 \text { included/ } \\
206 \text { completed } \\
66 \text { male, } 140 \text { female } \\
\text { Aged } 18-70 \text { years }\end{array}$ & Semiocclusive & $\begin{array}{l}\text { Induction phase: two transient low-level } \pm \text { reactions in one } \\
\text { subject (readings I, 2); three transient low-level } \pm \text { reactions } \\
\text { in one subject (readings 7-9) } \\
\text { Challenge: no reactions } \\
\text { Conclusion: no potential for dermal irritation or sensitization }\end{array}$ \\
\hline $\begin{array}{l}\text { Cleansing } \\
\text { lotion }\end{array}$ & $\begin{array}{l}\text { February-April 2000, } \\
\text { US }\end{array}$ & $\begin{array}{l}213 \text { included } / \\
183 \text { completed } \\
48 \text { male, } 135 \text { female } \\
\text { Aged } 18-69 \text { years }\end{array}$ & Occlusive & $\begin{array}{l}\text { Induction phase: one transient low-level } \pm \text { reaction in two } \\
\text { subjects (readings } 6,8) \text {; two transient low-level } \pm \text { reactions } \\
\text { in two subjects (readings } 4,5) \text {; four low level transient } \\
\text { reactions }(I \times I ; 3 \times \pm \text { ) in I subject (readings } 2-5 \text { ) } \\
\text { Challenge phase: one transient low-level reaction }( \pm \text { ) in four } \\
\text { subjects ( } 24 \text { hours, } 3 \times 48 \text { hours); two transient low-level } \\
\text { reactions }(I ; \pm \text { ) in one subject ( } 48 \text { hours, } 72 \text { hours) } \\
\text { Conclusion: no potential for dermal irritation or sensitization }\end{array}$ \\
\hline Cream & $\begin{array}{l}\text { December 2005- } \\
\text { January 2006, US }\end{array}$ & $\begin{array}{l}240 \text { included/ } \\
224 \text { completed } \\
59 \text { male, } 165 \text { female } \\
\text { Aged } 18-69 \text { years }\end{array}$ & Occlusive & $\begin{array}{l}\text { No reaction during induction phase } \\
\text { Challenge phase: one transient low-level reaction }( \pm \text { ) in one } \\
\text { subject ( } 48 \text { hours); two transient low-level } \pm \text { reactions in one } \\
\text { subject ( } 48 \text { hours, } 72 \text { hours) } \\
\text { Conclusion: no potential for dermal irritation or sensitization }\end{array}$ \\
\hline Night cream & $\begin{array}{l}\text { July-August 2006, } \\
\text { US }\end{array}$ & $\begin{array}{l}240 \text { included/ } \\
217 \text { completed } \\
68 \text { male, } 149 \text { female } \\
\text { Aged } 18-70 \text { years }\end{array}$ & Semiocclusive & $\begin{array}{l}\text { Induction phase: one transient low-level } \pm \text { reaction in two } \\
\text { subjects (readings } 2 \text { ) } \\
\text { Challenge phase: two transient low-level } \pm \text { reactions in one } \\
\text { subject ( } 48 \text { hours, } 72 \text { hours) } \\
\text { Conclusion: no potential for dermal irritation or sensitization }\end{array}$ \\
\hline Serum & $\begin{array}{l}\text { July-August 2006, } \\
\text { US }\end{array}$ & $\begin{array}{l}240 \text { included/ } \\
217 \text { completed } \\
68 \text { male, } 149 \text { female } \\
\text { Aged } 18-70 \text { years }\end{array}$ & Semiocclusive & $\begin{array}{l}\text { Induction phase: one transient low-level } \pm \text { reaction in three } \\
\text { subjects (readings } 2,9,9) \text {; one transient low-level reaction (I) } \\
\text { in one subject (reading } 5) \text {; two transient low-level reactions } \\
(I ; \pm) \text { in one subject (readings } 5,6) \\
\text { Challenge phase: one level I + edema reaction ( } 48 \text { hours), } \\
\text { two low-level transient reactions }(I) \text { in one subject } \\
(24 \text { hours, } 72 \text { hours); two transient low-level reactions } \\
(I ; \pm) \text { in one subject ( } 48 \text { hours, } 72 \text { hours) } \\
\text { Remark: test material did induce an edematous reaction } \\
\text { indicative of dermal sensitization in one human subject; } \\
\text { reaction not confirmed by a second patch testing } \\
\text { Conclusion: no potential of the product for dermal sensitization }\end{array}$ \\
\hline Baby cream & $\begin{array}{l}\text { February-March 2009, } \\
\text { Romania }\end{array}$ & $\begin{array}{l}\text { II } 4 \text { included/ } \\
109 \text { completed } \\
\text { I } 3 \text { male, } 96 \text { female } \\
\text { Aged } 18-70 \text { years }\end{array}$ & Semiocclusive & $\begin{array}{l}\text { Induction phase: one mild erythema }(I) \text { in one subject } \\
\text { (reading } 3)^{\prime} \\
\text { Challenge phase: no reaction } \\
\text { Conclusion: no potential for dermal irritation or sensitization }\end{array}$ \\
\hline
\end{tabular}


Table 2 (Continued)

\begin{tabular}{|c|c|c|c|c|}
\hline Test material & Date/country & Participants & Application & Results \\
\hline Handcream & $\begin{array}{l}\text { May-June 2002, } \\
\text { US }\end{array}$ & $\begin{array}{l}240 \text { included/ } \\
201 \text { completed } \\
59 \text { male, } 142 \text { female } \\
\text { Aged } 18-70 \text { years }\end{array}$ & Semiocclusive & $\begin{array}{l}\text { Induction phase: two transient low-level reactions }(I ; \pm) \\
\text { in one subject (readings } 3,4 \text { ); eight low-level reactions }( \pm) \\
\text { in one subject (readings } 2-9 \text { ) } \\
\text { Challenge phase: one transient low-level reaction }( \pm) \\
\text { in one subject ( } 72 \text { hours); three level I + edema reactions } \\
\text { in one subject ( } 48 \text { hours, } 72 \text { hours, } 96 \text { hours) } \\
\text { Remarks: test material did induce an edematous reaction } \\
\text { indicative of dermal sensitization in one human subject; } \\
\text { reaction confirmed with the finished product by a second } \\
\text { patch testing but not with Avena sativa } \\
\text { Conclusion: doubtful }\end{array}$ \\
\hline $\begin{array}{l}\text { Exfoliating } \\
\text { cleanser }\end{array}$ & $\begin{array}{l}\text { March-May 2009, } \\
\text { Romania }\end{array}$ & $\begin{array}{l}\text { I } 4 \text { included/ } \\
\text { 109 completed } \\
23 \text { male, } 86 \text { female } \\
\text { Aged I8-68 years }\end{array}$ & $\begin{array}{l}2 \% \text { dilution, } \\
\text { semiocclusive }\end{array}$ & $\begin{array}{l}\text { No reaction during induction phase or challenge phase } \\
\text { Conclusion: no potential for dermal irritation or sensitization }\end{array}$ \\
\hline $\begin{array}{l}\text { Wash } \\
\text { (head-to-toe) }\end{array}$ & $\begin{array}{l}\text { August- } \\
\text { September 2007, } \\
\text { US }\end{array}$ & $\begin{array}{l}245 \text { included/ } \\
216 \text { completed } \\
59 \text { male, } 157 \text { female } \\
\text { Aged I8-70 years }\end{array}$ & $\begin{array}{l}8 \% \text { dilution, } \\
\text { occlusive }\end{array}$ & $\begin{array}{l}\text { Induction phase: one transient low-level } \pm \text { reaction in three } \\
\text { subjects (readings } 2,7,7) \text {; one transient low-level reaction } \\
\text { (I) in one subject (reading } 2 \text { ); two transient low-level } \\
\text { reactions }(I ; \pm \text { ) in one subject (readings } 7,8) \\
\text { Challenge phase: two transient low-level reactions }(I ; \pm \text { ) in } \\
\text { two subjects ( } 48 \text { hours, } 72 \text { hours); three transient } \\
\text { low-level reactions }(2 \times I ; I \times \pm \text { ) in one subject } \\
\text { ( } 48 \text { hours, } 72 \text { hours, } 96 \text { hours) } \\
\text { Conclusion: no potential for dermal irritation or sensitization }\end{array}$ \\
\hline
\end{tabular}

Note: 'Note that different scoring methods were used at the two study sites.

other subject, reactions were confirmed for the complete product.

A total of 645 subjects were enrolled in the 12 safety-in-use studies, which were completed by 615 subjects. Seven studies tested skin reactions to different facial, body, and hair cleansers as well as creams in female subjects with normal to very dry skin and skin sensitivity ranging from not sensitive to very sensitive by means of subjective self-evaluation (UK studies, Table 3). Among the 402 subjects who returned their questionnaires, 18 reported adverse reactions. The majority of these reactions (nine) were moderate in nature, followed by slight (seven) and severe (two) reactions. In another study, the reaction to a facial cleanser was evaluated in female subjects with normal to oily or dry skin, of whom $32 \%$ had a history of atopic dermatitis. No reaction was reported by the investigating dermatologist. Two studies evaluated the ocular tolerance of a facial cleanser in 43 female subjects with normally sensitive eyes. Eye reactions were documented in three of the subjects and confirmed by clinical ophthalmological evaluation, and in nine subjects according to self-evaluation (Table 4). Finally, two studies tested the reactions to two test materials in 80 adults and 30 children with normal to dry skin. A history of atopic dermatitis was reported for 27 of 80 adults and for 11 of 30 children who participated in these studies. For adult subjects, the application period was followed by a patch test using the diluted or undiluted product. No clinical reaction was observed during the application period by any of the 110 subjects nor was there a reaction after patch testing in the 80 adults.

\section{Efficacy analysis}

A total of 47 subjects completed the study of the clinical efficacy of an oatmeal-containing body cream, with evaluation on day 14, and 46 subjects underwent evaluation on days 28 and 42 .

\section{Skin hydration}

On the forearm, skin hydration was significantly higher at all time points, including at 2 weeks after cessation of application (day 42), compared with baseline. For the leg area, an increase in hydration was observed that was significantly higher than on the control site at all time points (Figure 1).

\section{Desquamation index and dead cell surface area}

The surface area of dead epithelial cells and the epithelial desquamation index on the forearm were significantly reduced at all assessment time points compared with baseline. On the leg, a reduction in the dead cell surface 
Table 3 Summary of safety-in-use studies

\begin{tabular}{|c|c|c|c|c|}
\hline \multirow{2}{*}{$\begin{array}{l}\text { Test } \\
\text { material }\end{array}$} & \multirow[t]{2}{*}{ Date/country } & \multirow{2}{*}{$\begin{array}{l}\text { Participants } \\
\text { Skin/hair type' and skin/eye } \\
\text { sensitivity' (if applicable) }\end{array}$} & \multirow[t]{2}{*}{ Application } & \multirow{2}{*}{$\begin{array}{l}\text { Result (number of subjects } \\
\text { with reaction/total } \\
\text { number of subjects) }\end{array}$} \\
\hline & & & & \\
\hline $\begin{array}{l}\text { Shower and } \\
\text { bath oil }\end{array}$ & $\begin{array}{l}\text { December 2006, } \\
\text { UK }\end{array}$ & $\begin{array}{l}60 \text { included (dry, very dry body skin), } \\
53 \text { completed (skin sensitivity: } \\
19 \% \text { not sensitive, } 47 \% \text { a little sensitive, } \\
23 \% \text { sensitive, II\% very sensitive) } \\
\text { Aged } 18-55 \text { years } \\
\text { Female }\end{array}$ & $\begin{array}{l}\text { Use product on seven } \\
\text { consecutive days instead } \\
\text { of usual shower product }\end{array}$ & $\begin{array}{l}\text { Adverse reaction: } 3.8 \% \\
2 / 53 \text { (one moderate, one slight) }\end{array}$ \\
\hline $\begin{array}{l}\text { Cream } \\
\text { moisturizing } \\
\text { oil }\end{array}$ & $\begin{array}{l}\text { December 2006, } \\
\text { UK }\end{array}$ & $\begin{array}{l}60 \text { included (dry, normal to dry body skin), } \\
56 \text { completed (skin sensitivity: } \\
23 \% \text { not sensitive, } 52 \% \text { a little sensitive, } \\
21 \% \text { sensitive, } 4 \% \text { very sensitive) } \\
\text { Aged } 18-55 \text { years } \\
\text { Female }\end{array}$ & $\begin{array}{l}\text { Use product once a } \\
\text { day on seven } \\
\text { consecutive days } \\
\text { instead of usual } \\
\text { body moisturizer }\end{array}$ & $\begin{array}{l}\text { Adverse reaction: } 3.6 \% \\
\text { Two of } 56 \\
\text { (one severe, one moderate) }\end{array}$ \\
\hline Shower gel & $\begin{array}{l}\text { August 2006, } \\
\text { UK }\end{array}$ & $\begin{array}{l}60 \text { included (dry, sensitive body skin), } \\
59 \text { completed (skin sensitivity } \\
\text { not indicated) } \\
\text { Aged } 20-50 \text { years } \\
\text { Female }\end{array}$ & $\begin{array}{l}\text { Use product on seven } \\
\text { consecutive days } \\
\text { instead of usual } \\
\text { shower product }\end{array}$ & $\begin{array}{l}\text { Adverse reaction: } 3.4 \% \\
\text { Two of } 59 \text { (two moderate) }\end{array}$ \\
\hline Night cream & $\begin{array}{l}\text { April-May 2009, } \\
\text { UK }\end{array}$ & $\begin{array}{l}70 \text { included (facial skin: normal, } \\
\text { dry, normal to dry, normal to greasy, } \\
\text { normal/dry/greasy) } \\
64 \text { completed (skin sensitivity: } \\
5 \% \text { not sensitive, } 61 \% \text { a little sensitive, } \\
30 \% \text { sensitive, } 5 \% \text { very sensitive) } \\
\text { Aged } 25-49 \text { years } \\
\text { Female }\end{array}$ & $\begin{array}{l}\text { Use product on } \\
28 \text { consecutive days } \\
\text { instead of usual } \\
\text { night-time moisturizer }\end{array}$ & $\begin{array}{l}\text { Adverse reaction: } 10.9 \% \\
\text { Seven of } 64 \text { (five subjects with } \\
\text { slight to moderate reactions, } \\
\text { one subject with moderate to } \\
\text { severe reactions, and one } \\
\text { subject with severe reactions) }\end{array}$ \\
\hline $\begin{array}{l}\text { Conditioning } \\
\text { shampoo }\end{array}$ & $\begin{array}{l}\text { January- } \\
\text { February 2007, UK }\end{array}$ & $\begin{array}{l}60 \text { included (all hair types) } \\
\text { Male } 30 \text {, female } 30 \\
55 \text { completed } \\
\text { Aged } 18-55 \text { years }\end{array}$ & $\begin{array}{l}\text { Use product on } \\
10 \text { occasions, } \\
\text { no use of conditioner }\end{array}$ & $\begin{array}{l}\text { Adverse reaction: } 3.6 \% \\
\text { Two of } 55 \text { (two moderate) }\end{array}$ \\
\hline Body lotion & $\begin{array}{l}\text { November- } \\
\text { December 2006, UK }\end{array}$ & $\begin{array}{l}60 \text { included (dry, normal to dry body skin), } \\
57 \text { completed (skin sensitivity: } \\
12 \% \text { not sensitive, } 39 \% \text { a little sensitive, } \\
19 \% \text { sensitive, } 30 \% \text { very sensitive) } \\
\text { Aged } 18-55 \text { years } \\
\text { Female }\end{array}$ & $\begin{array}{l}\text { Use product on seven } \\
\text { consecutive days as } \\
\text { frequently as required }\end{array}$ & Adverse reaction: $0 \%$ \\
\hline $\begin{array}{l}\text { Liquid hand } \\
\text { wash }\end{array}$ & $\begin{array}{l}\text { October 2006, } \\
\text { UK }\end{array}$ & $\begin{array}{l}60 \text { included (dry, normal to dry, very dry } \\
\text { hand skin), } 58 \text { completed (skin sensitivity: } \\
12 \% \text { not sensitive, } 55 \% \text { a little sensitive, } \\
22 \% \text { sensitive, } 10 \% \text { very sensitive) } \\
\text { Aged } 18-55 \text { years } \\
\text { Female }\end{array}$ & $\begin{array}{l}\text { Use product on seven } \\
\text { consecutive days as } \\
\text { frequently as required } \\
\text { instead of usual hand } \\
\text { wash product }\end{array}$ & $\begin{array}{l}\text { Adverse reaction: } 5.2 \% \\
\text { Three of } 58 \text { (one slight and } \\
\text { two moderate) }\end{array}$ \\
\hline $\begin{array}{l}\text { Facial } \\
\text { exfoliating } \\
\text { cleanser }\end{array}$ & $\begin{array}{l}\text { March-April 2009, } \\
\text { Bulgaria }\end{array}$ & $\begin{array}{l}62 \text { included (normal, mixed oily, oily, } \\
\text { mixed dry, dry skin), } 60 \text { completed } \\
\text { (sensitive skin 100\%, history of } \\
\text { atopy } 32 \% \text { ), two withdrew consent } \\
\text { Aged } 18-60 \text { years } \\
\text { Female }\end{array}$ & $\begin{array}{l}\text { Use product } I \times \text { per day } \\
\text { on face and neck during } \\
3 \text { weeks }\end{array}$ & $\begin{array}{l}\text { Safety evaluation: } \\
\text { Adverse reactions observed } \\
\text { by dermatologist: } 0 / 60 \\
\text { Adverse reaction reported } \\
\text { by subjects: } 3 / 60\end{array}$ \\
\hline $\begin{array}{l}\text { Intimate } \\
\text { wash }\end{array}$ & $\begin{array}{l}\text { January 2007, } \\
\text { Germany }\end{array}$ & $\begin{array}{l}60 \text { included ( } 48 \% \text { healthy skin, } \\
17 \% \text { dry skin, } 2 \% \text { sensitive skin, } \\
33 \% \text { atopic dermatitis/eczema-free } \\
\text { interval), } 60 \text { completed } \\
\text { Aged I8-58 years } \\
\text { Female }\end{array}$ & $\begin{array}{l}\text { Use product at least } \\
I \times \text { per day during } \\
4 \text { weeks } \\
\text { Subsequent occlusive } \\
\text { patch test with } \\
1 \%, 2 \%, 5 \% \text { dilutions, } \\
\text { inner forearm for } \\
24 \text { hours }\end{array}$ & $\begin{array}{l}\text { After } 4 \text { weeks: adverse reaction: } 0 \\
\text { Patch test: no reaction at any } \\
\text { concentration }\end{array}$ \\
\hline
\end{tabular}


Table 3 (Continued)

\begin{tabular}{|c|c|c|c|c|}
\hline \multirow{2}{*}{$\begin{array}{l}\text { Test } \\
\text { material }\end{array}$} & \multirow[t]{2}{*}{ Date/country } & Participants & \multirow[t]{2}{*}{ Application } & \multirow{2}{*}{$\begin{array}{l}\text { Result (number of subjects } \\
\text { with reaction/total } \\
\text { number of subjects) }\end{array}$} \\
\hline & & $\begin{array}{l}\text { Skin/hair type' and skin/eye } \\
\text { sensitivity' (if applicable) }\end{array}$ & & \\
\hline Baby milk & $\begin{array}{l}\text { January } 2007, \\
\text { Germany }\end{array}$ & $\begin{array}{l}20 \text { adults included ( } 25 \% \text { normal skin, } \\
20 \% \text { dry skin, } 20 \% \text { sensitive skin, } \\
35 \% \text { atopic dermatitis/eczema free interval), } \\
20 \text { completed } \\
\text { Aged } 21-47 \text { years } \\
\text { Six male, } 14 \text { female } \\
30 \text { children included ( } 27 \% \text { normal skin, } \\
20 \% \text { dry skin, } 17 \% \text { sensitive skin, } \\
37 \% \text { atopic dermatitis/eczema free interval), } \\
30 \text { completed } \\
\text { Aged } 8 \text { months to } 4 \text { years } \\
\text { II male, } 19 \text { female }\end{array}$ & $\begin{array}{l}\text { Use product at least } \\
2 \times \text { per day during } \\
4 \text { weeks } \\
\text { Subsequent occlusive } \\
\text { patch test with adults } \\
\text { only (undiluted), inner } \\
\text { forearm for } 24 \text { hours }\end{array}$ & $\begin{array}{l}\text { After } 4 \text { weeks: adverse reaction: } 0 \\
\text { Patch test: no reaction }\end{array}$ \\
\hline
\end{tabular}

Note: 'According to information supplied by subject.

area was observed that was significantly larger than that on the control area at all time points. In this area, the desquamation index also diminished to a significantly greater extent than in the control area during the application period (Figure 2).

\section{Clinical evaluation}

Throughout the application period, all parameters (skin dryness, appearance of squamae, and skin roughness) assessed by the dermatologist on the leg were significantly more improved than on the control area (Figure 3). This was still the case beyond cessation of treatment on day 42 . No clinically significant adverse reactions were noted during the course of the study.

\section{Self-evaluation}

At days 14 and $28,63 \%-100 \%$ of the subjects responded favorably ("agree" or "rather agree") to a series of questions concerning subjective evaluation of signs of skin dryness after application of the product (Figure 4). Two weeks after cessation of application, the proportion of positive responses was $40 \%-61 \%$.

\section{Discussion}

We tested the irritation and sensitization potential of 12 oatmeal-containing personal care products in 2291 subjects as part of a series of repeat insult patch testing studies. We observed only low-level reactions corresponding to faint erythema, minimal erythema, or obvious erythema according to the scoring system used. All of the subjects concerned (23/2291, ie, $1.0 \%)$ experienced transient reactions, with the exception of one individual with persistent erythema. These data indicate a very low irritancy potential for the study materials. In the subsequent challenge period, we saw mostly transient low-level reactions (14/2291 subjects, ie, $0.6 \%$ ). Three subjects had a low-level reaction accompanied by edema. One of these subjects had a persistent reaction on three consecutive readings. However, this reaction was doubtful because it was confirmed with the complete product and not with $A$. sativa. The test material in that case was a

Table 4 Summary of ocular tolerability testing under ophthalmological control (safety-in-use studies)

\begin{tabular}{|c|c|c|c|c|}
\hline Test material & Date/country & $\begin{array}{l}\text { Participants } \\
\text { Skin/hair type' and skin/eye } \\
\text { sensitivity' (if applicable) }\end{array}$ & Application & $\begin{array}{l}\text { Result (number of subjects } \\
\text { with reaction/per total } \\
\text { number of subjects) }\end{array}$ \\
\hline $\begin{array}{l}\text { Face and eye } \\
\text { cleansing lotion }\end{array}$ & $\begin{array}{l}\text { September 2009, } \\
\text { Poland }\end{array}$ & $\begin{array}{l}22 \text { included (normally sensitive eyes), } \\
22 \text { completed } \\
\text { Aged } 18-70 \text { years } \\
\text { Female }\end{array}$ & $\begin{array}{l}\text { Use product } 2 \times \text { per day } \\
\text { on face including eye area } \\
\text { and neck during } 3 \text { weeks }\end{array}$ & Clinical signs: $0 \%$ \\
\hline $\begin{array}{l}\text { Face and eye } \\
\text { cleansing lotion }\end{array}$ & $\begin{array}{l}\text { September 2009, } \\
\text { Poland }\end{array}$ & $\begin{array}{l}22 \text { included (normally sensitive eyes), } \\
21 \text { completed } \\
\text { Aged } 18-60 \text { years } \\
\text { Female }\end{array}$ & $\begin{array}{l}\text { Use product } 2 \times \text { per day } \\
\text { on face including eye area } \\
\text { and neck for } 3 \text { weeks }\end{array}$ & $\begin{array}{l}\text { Clinical signs: I } 4 \% \\
3 / 2 \text { I (possibly attributable to product } \\
\text { and for two subjects only on one eye) }\end{array}$ \\
\hline
\end{tabular}


A

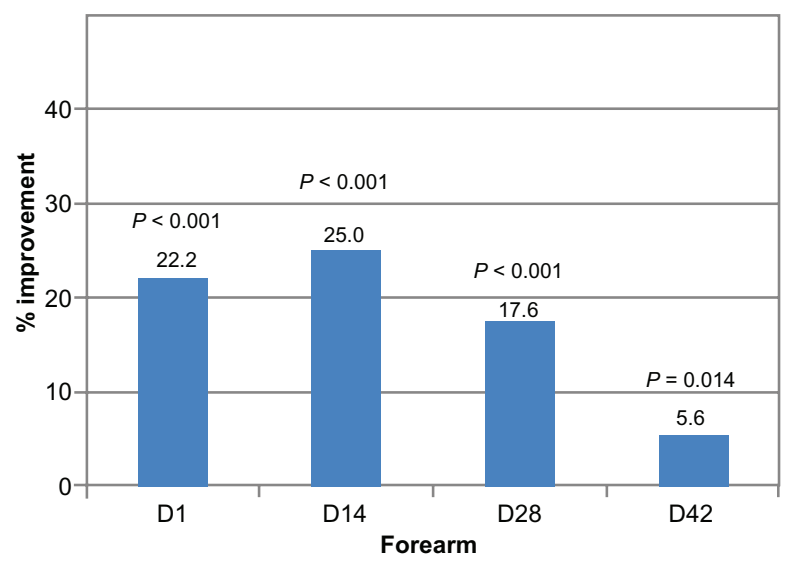

B

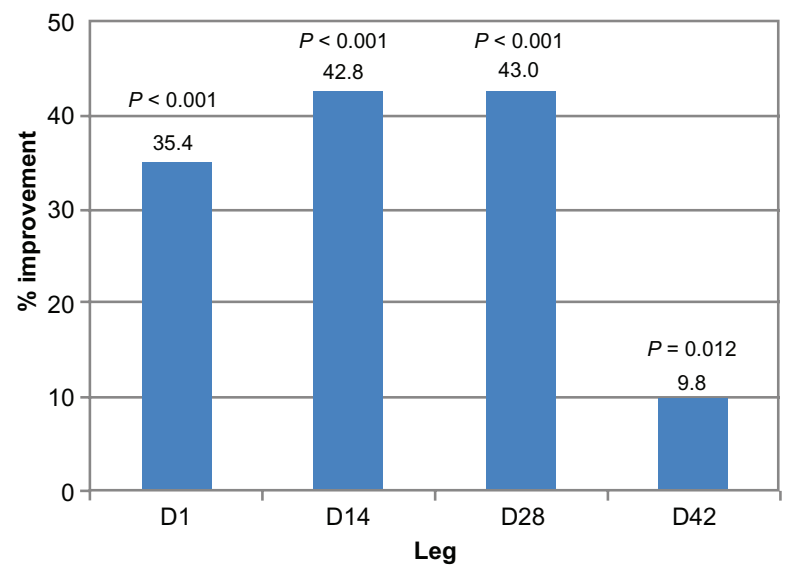

Figure I Skin hydration increases during and after use of oatmeal-containing cream.

Notes: Hydration of the forearm increased significantly during the application period (days I-28) and afterwards (day 42) compared with baseline. Hydration of the leg increased significantly more compared with the control area at all time points.
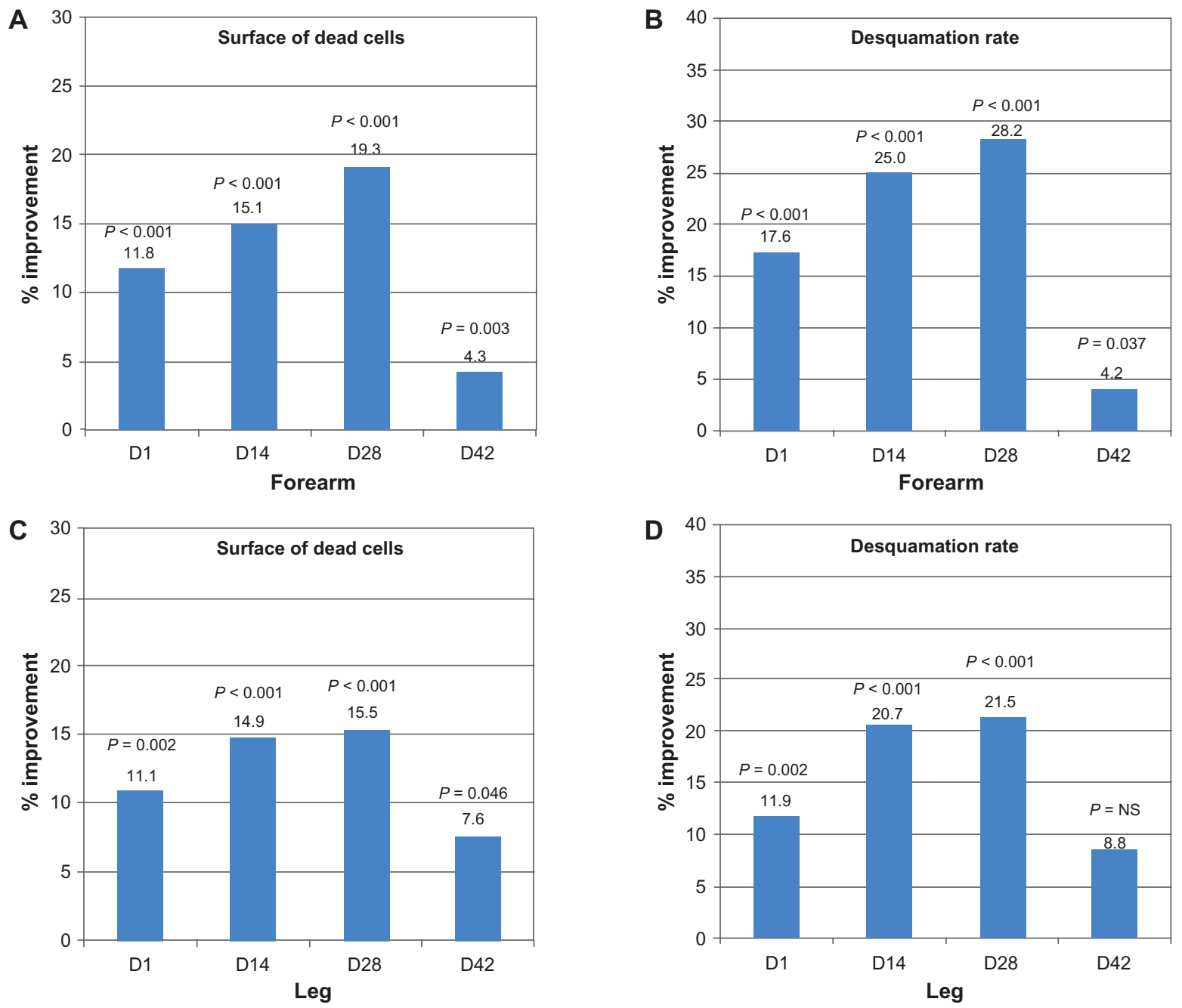

Figure 2 Surface area of dead cells and desquamation index diminish with use of oatmeal-containing cream. Surface of dead cells (A) and desquamation rate (B) were compared with baseline for the forearm area and their reduction is indicated as percentage improvement. Data derived from leg measurements was compared with the control area for surface of dead cells (C) and desquamation rate (D).

Abbreviation: NS, not statistically significant. 


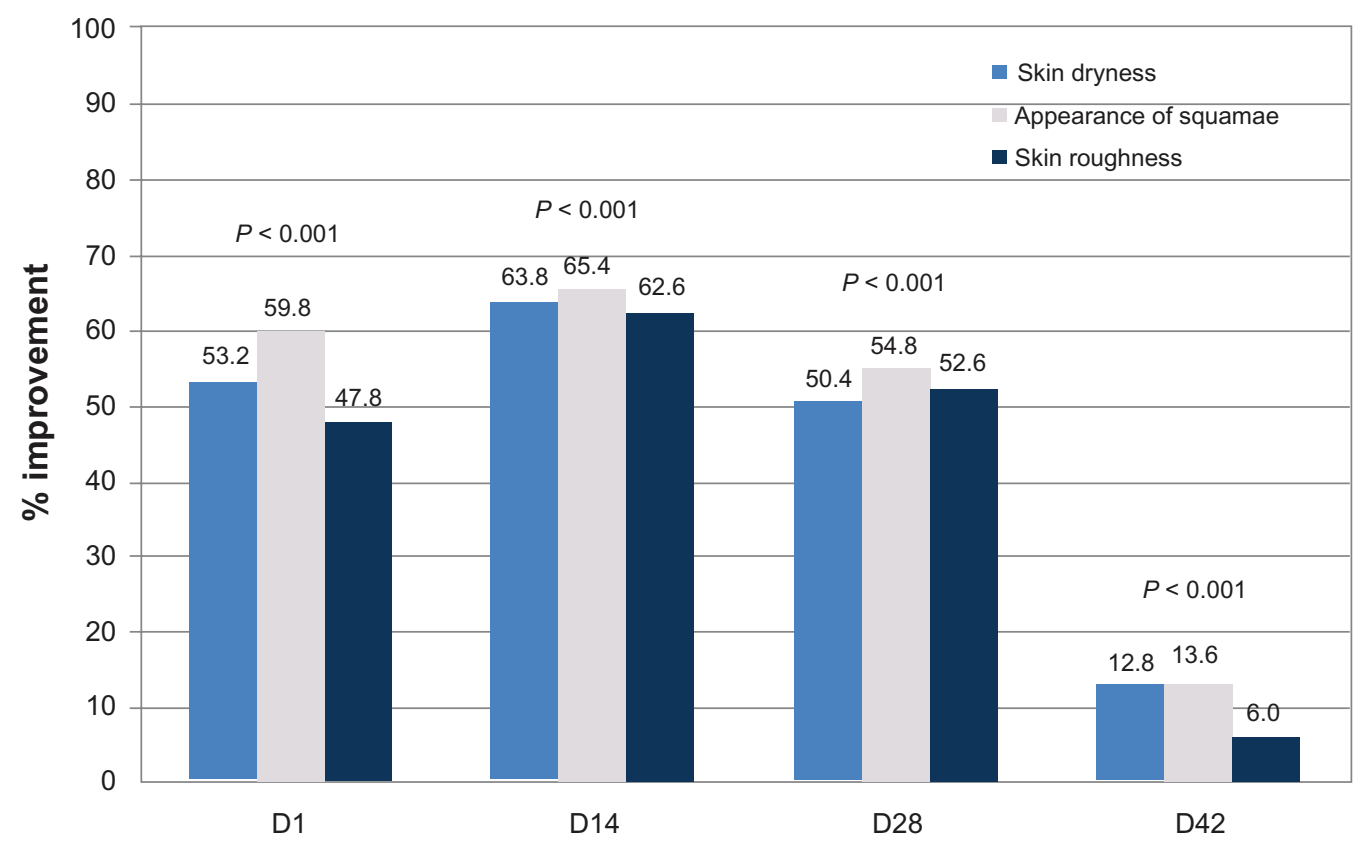

Figure 3 Clinically assessed parameters improve with use of oatmeal-containing cream.

Note: At all time points, the three parameters assessed, ie, skin dryness, appearance of squamae, and skin roughness, were significantly more improved on the treated leg area compared with the control area.

hand cream. The edematous reaction was transient in the two other subjects.

Furthermore, we performed safety-in-use testing of both "leave-on" (creams and lotions) and "wash/rinseoff" (shower oils and shower gels, shampoo, liquid hand wash, facial cleansers, intimate wash) oatmeal-containing products to assess their irritancy potential. The majority of these safety-in-use studies (8/12) included subjects with self-reported sensitive skin. Three studies also included subjects with a self-reported history of atopic dermatitis. The prevalence of sensitive skin among the subjects was $77 \%-100 \%$, but was lower in two of the studies including

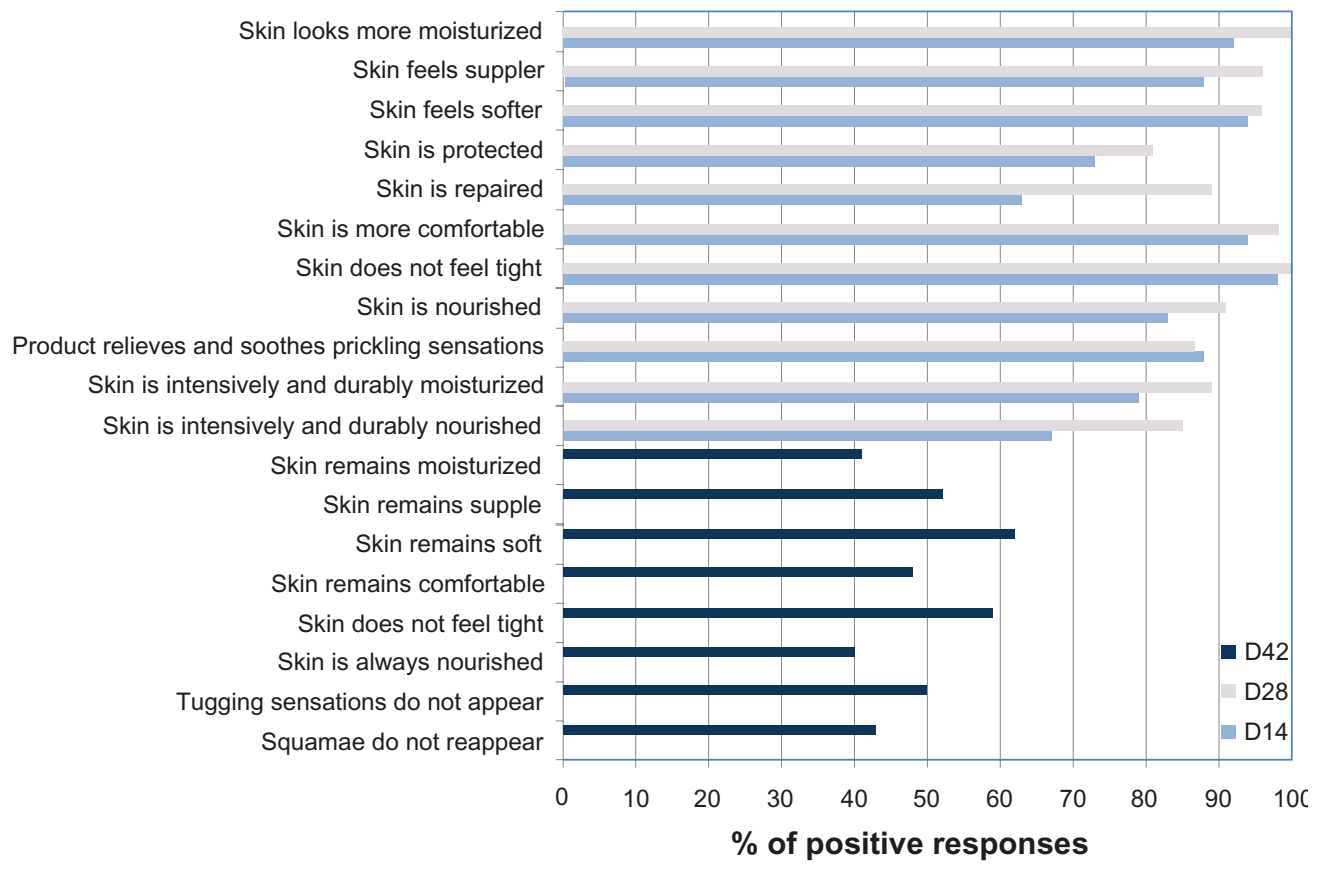

Figure 4 Subjective evaluation of effect on signs of skin dryness.

Note: The percentage of patients who reported a positive effect on skin dryness is depicted (combination of answers "agree" and "rather agree"). 
atopic subjects $(2 \%-20 \%)$. The proportion of subjects with sensitive skin in our studies was higher than that in the general population, as indicated by a frequency of self-reported sensitive skin in a random population sample in the UK of $51 \%$ in women and $38 \%$ in men and of $52 \%$ in women in the US. ${ }^{5,6}$ Of the tests involving self-assessed skin reactions, the highest percentage of reactions was observed for a leave-on night cream, which provoked reactions in $7 / 64$ subjects (10.9\%), followed by a face and eye cleanser (two of 22 subjects, 9.1\%). Other test materials (mainly wash/ rinse-off products) had a lower frequency of adverse reactions ( $0 \%$ for a face and eye cleanser and a body lotion, $<4 \%$ for shower oils, gels, and a shampoo, $5 \%$ for a facial exfoliating cleanser, and 5.2\% for a liquid hand wash). In one of the two studies including ophthalmological evaluation after use of a face and eye cleansing lotion, we observed $14 \%$ of clinical eye signs with possible implication of the product in one case and reactions only on one eye in two subjects.

For two studies, in which one third of subjects were atopic, we did not find any clinical signs of skin irritation in either adults or infants or when assessed, of allergic sensitization in adults. Atopic dermatitis is an inflammatory skin condition particularly affecting infants and children. It appears to be increasing in prevalence, ${ }^{12,13}$ and affects $10 \%-20 \%$ of individuals in the first decade of life. ${ }^{14}$ In a recent study of 67 children with atopic dermatitis, it was suggested that use of moisturizers containing oat protein is a risk factor for oat sensitization. ${ }^{8}$ In the same study, $15 \%$ of 302 children aged 4 months to 15 years with atopic dermatitis had a positive oat extract atopy patch test result and $19 \%$ had a positive skin prick test result. However, the frequency of oat sensitivity was much lower in another study performed in 202 atopic children, with sensitivity reported in $2.9 \%$ of children who were oat cream users and in $2.1 \%$ of those who had never used oat cream. ${ }^{9}$ No sensitization to topical colloidal oatmeal was found in a randomized, double-blind study performed in 65 atopic and nonatopic children between 6 months and 2 years of age. ${ }^{10}$ In our studies, we limited patch tests after in-use application of the test material to adults, and did not observe any allergic sensitization in 80 participants.

The skin hydrating properties of colloidal oatmeal have been ascribed to its propensity to form an occlusive film capable of binding water in the stratum corneum. ${ }^{1}$ We observed a significant moisturizing effect of an oatmeal-containing cream on dry skin throughout the application period, which was sustained for 2 weeks afterwards. This was indicated by increased hydration, a reduced desquamation index, and a reduced surface area occupied by dead skin cells, as well as by clinical evaluation and subjective self-evaluation.

\section{Conclusion}

We demonstrated that the irritation and allergenic potential of a diverse range of oatmeal-containing personal care products is low. With the exception of one subject, in whom the reaction to A. sativa was doubtful, more than 2300 subjects did not show allergic sensitization. Moreover, in allergic patients we reported by consumers of 445,820 products sold during a 3 -year period. In addition, we found a sustainable moisturizing effect of oatmeal-containing products on dry skin.

\section{Acknowledgment}

The authors thank Beate Gerstbrein for her help in preparation of the manuscript.

\section{Disclosure}

Vincent Walczak and Judith Nebus, both employees of Johnson \& Johnson CPWW, provided information on the studies mentioned in this paper.

\section{References}

1. Kurtz ES, Wallo W. Colloidal oatmeal: history, chemistry and clinical properties. J Drugs Dermatol. 2007;6(2):167-170.

2. Wu J. Anti-inflammatory ingredients. J Drugs Dermatol. 2008; 7(Suppl 7):S13-S16.

3. Grimalt R, Mengeaud V, Cambazard F. The steroid-sparing effect of an emollient therapy in infants with atopic dermatitis: a randomized controlled study. Dermatology. 2007;214(1):61-67.

4. Sur R, Nigam A, Grote D, Liebel F, Southall MD. Avenanthramides, polyphenols from oats, exhibit anti-inflammatory and anti-itch activity. Arch Dermatol Res. 2008;300(10):569-574.

5. Jourdain R, de Lacharriere O, Bastien P, Maibach HI. Ethnic variations in self-perceived sensitive skin: epidemiological survey. Contact Dermatitis. 2002;46(3):162-169.

6. Willis CM, Shaw S, De Lacharriere O, et al. Sensitive skin: an epidemiological study. Br J Dermatol. 2001;145(2):258-263.

7. Adams RM, Maibach HI. A five-year study of cosmetic reactions. J Am Acad Dermatol. 1985;13(6):1062-1069.

8. Boussault P, Leaute-Labreze C, Saubusse E, et al. Oat sensitization in children with atopic dermatitis: prevalence, risks and associated factors. Allergy. 2007;62(11):1251-1256.

9. Rancé F, Dargassies J, Dupuy P, Schmitt AM, Guerin L, Dutau G. Faut-il contre-indiquer l'utilisation des émollients à base d'avoine chez l'enfant atopique? (Are there contra-indications in the use of oatmealbased emollients in atopic children?) Rev Fr Allergol Immunol Clin. 2001;41:477-483. French.

10. Pigatto P, Bigardi A, Caputo R, et al. An evaluation of the allergic contact dermatitis potential of colloidal grain suspensions. Am J Contact Dermat. 1997;8(4):207-209.

11. Fisher AA. Contact Dermatitis. Philadelphia, PA: Lea and Febiger;1986.

12. Schultz Larsen F, Diepgen T, Svensson A. The occurrence of atopic dermatitis in north Europe: an international questionnaire study. $\mathrm{J} \mathrm{Am}$ Acad Dermatol. 1996;34(5 Pt 1):760-764.

13. Spergel JM, Paller AS. Atopic dermatitis and the atopic march. JAllergy Clin Immunol. 2003;112(6 Suppl):S118-S127.

14. Krakowski AC, Eichenfield LF, Dohil MA. Management of atopic dermatitis in the pediatric population. Pediatrics. 2008;122(4):812-824. 


\section{Publish your work in this journal}

Clinical, Cosmetic and Investigational Dermatology is an international, peer-reviewed, open access, online journal that focuses on the latest clinical and experimental research in all aspects of skin disease and cosmetic interventions. All areas of dermatology will be covered; contributions will be welcomed from all clinicians and basic science researchers globally. This journal is indexed on CAS. The manuscript management system is completely online and includes a very quick and fair peer-review system, which is all easy to use. Visit http://www.dovepress.com/testimonials.php to read real quotes from published authors.

Submit your manuscript here: http://www.dovepress.com/clinical-cosmetic-and-investigational-dermatology-journal 